\title{
Carotid-vertebral artery bypass with saphenous vein graft for symptomatic vertebrobasilar insufficiency
}

\author{
*Hongzhou Duan, MD,' Dapeng Mo, MD, ${ }^{1,2}$ Yang Zhang, MD, ${ }^{1}$ Jiayong Zhang, MD,1 and \\ Liang Li, MD' \\ 1Department of Neurosurgery, Peking University First Hospital; and 'Department of the Interventional Neuroradiology, Beijing \\ Tiantan Hospital Affiliated to the Capital Medical University, Beijing, China
}

OBJECTIVE Symptomatic steno-occlusion of the proximal vertebral artery (VA) or subclavian artery (ScA) heralds a poor prognosis and high risk of stroke recurrence despite medical therapy, including antiplatelet or anticoagulant drugs. In some cases, the $V_{2}$ segment of the cervical VA is patent and perfused via collateral vessels. The authors describe 7 patients who were successfully treated by external carotid artery (ECA)-saphenous vein (SV)-VA bypass.

METHODS Seven cases involving symptomatic patients were retrospectively studied: 3 cases of $V_{1}$ segment occlusion, 2 cases of severe in-stent restenosis in the $V_{1}$ segment, and 2 cases of occlusion of the proximal ScA. All patients underwent ECA-SV-VA bypass. The ECA was isolated and retracted, and the anterior wall of the transverse foramen was unroofed. The VA was exposed, and then the 2 ends of the SV were anastomosed to the VA and ECA in an end-to-side fashion.

RESULTS Surgical procedures were all performed as planned, with no intraoperative complications. There were 2 postoperative complications (severe laryngeal edema in one case and shoulder weakness in another), but both patients recovered fully and measures were taken to minimize laryngeal edema and its effects in subsequent cases. All patients experienced improvement of their symptoms. No new neurological deficits were reported. Postoperative angiography demonstrated that the anastomoses were all patent, and analysis of follow-up data (range of follow-up 12-78 months) revealed no further ischemic events in the vertebrobasilar territory.

CONCLUSIONS The ECA-SV-VA bypass is a useful treatment for patients who suffer medically refractory ischemic events in the vertebrobasilar territory when the proximal part of the VA or ScA is severely stenosed or occluded but the $\mathrm{V}_{2}$ segment of the cervical VA is patent.

https://thejns.org/doi/abs/10.3171/2018.11.FOCUS18360

KEYWORDS bypass; vertebrobasilar insufficiency; revascularization

$\mathrm{T}$ HE subclavian artery (ScA) and vertebral artery (VA) supply blood to vessels in the posterior circulation, and the steno-occlusion of the bilateral or dominant side of these arteries results in stroke in the vertebrobasilar territory. The atherosclerotic steno-occlusive changes in the VA generally occur in the $\mathrm{V}_{1}$ segment. The contralateral VA is sometimes occluded or hypoplastic, and in these cases the decreased blood flow in the chronically occluded dominant-side VA is compensated for by flow from collateral arteries from the anterior circle of Willis or extracranial arteries, such as the occipital artery (OA) and muscular branches. ${ }^{2}$ If the collateral blood supply is insufficient, patients will experience recurrent stroke or ischemic symptoms despite antiplatelet and/or anticoagulant medication. Angioplasty or stenting might be a promising treatment for stenosis at the origin of the VA, but the procedure is not suitable for occlusion or cases of in-stent stenosis. ${ }^{15,20}$ For such cases, many neurosurgeons recommend revascularization of the blood flow in the posterior circulation by using bypass surgery. Successful bypass procedures from the OA to the $\mathrm{V}_{3}$ segment of the VA, from the OA to the posterior inferior cerebellar artery (PICA), and from the OA to the anterior inferior cerebellar artery (AICA) have been reported. ${ }^{1,11,12}$ However, bypass that includes the OA

ABBREVIATIONS AICA = anterior inferior cerebellar artery; CCA = common carotid artery; ECA = external carotid artery; $\mathrm{mRS}=$ modified Rankin Scale; NIHSS = National Institutes of Health Stroke Scale; OA = occipital artery; PICA = posterior inferior cerebellar artery; POD = postoperative day; ScA = subclavian artery; SV = saphenous vein; $\mathrm{TCD}=$ transcranial Doppler sonography; VA = vertebral artery.

SUBMITTED July 16, 2018. ACCEPTED November 13, 2018.

INCLUDE WHEN CITING DOI: 10.3171/2018.11.FOCUS18360.

${ }^{*}$ H.D. and D.M. contributed equally to this article and share first authorship. 
as the donor artery provides low blood flow, and harvesting of the OA is difficult. Sometimes when the VA or the $\mathrm{ScA}$ is occluded at the origin or a severe stenosis exists in the VA stent, the distal portion of the cervical VA is patent and is often perfused via muscular branches or other collateral vessels. Therefore, a high-flow bypass from the external carotid artery (ECA) to the $\mathrm{V}_{2}$ segment of the VA should be considered. Here, we report on 7 patients with symptomatic stenosis or occlusion in the $\mathrm{V}_{1}$ segment or the proximal $\mathrm{ScA}$, who were successfully treated by ECA-VA bypass using an interposed saphenous vein (SV) graft.

\section{Methods \\ Patient Data}

Between January 2008 and December 2016, 7 patients with symptomatic VA or ScA steno-occlusion were treated with ECA-SV-VA bypass. The group included 5 men and 2 women; their ages ranged from 51 to 69 years (mean 62.14 years). Five patients also presented with hypertension, and 4 patients presented with diabetes. Two patients presented with coronary heart disease, and one of these patients underwent coronary artery bypass grafting. One patient presented with a paraclinoid carotid artery aneurysm, which was successfully treated by coil embolization. All patients suffered from ischemic symptoms of posterior cerebral circulation, such as vertigo, postural dizziness, ataxia, dysarthria, or hemiparesis. Fresh or old cerebellar and/or brainstem infarctions were confirmed by MRI in 5 patients. The patients' preoperative National Institutes of Health Stroke Scale (NIHSS) scores ranged from 1 to 16 (mean 5.43), and their preoperative modified Rankin Scale (mRS) scores ranged from 1 to 3 (mean 1.57).

\section{Treatment Procedure}

After the patients were admitted to the hospital, routine preoperative examinations were performed, including examinations of cardiac and pulmonary function. MRI was conducted to confirm infarction or insufficiency in the vertebrobasilar territory. Transcranial Doppler sonography (TCD) and cervical vascular ultrasound examinations were performed to evaluate the blood flow of the posterior circulation and confirm the patency of the $V_{2}$ segment of the VA. Preoperative cerebral angiography was performed in all cases, and collateral blood flow was evaluated. Aspirin treatment $(100 \mathrm{mg} /$ day) was started 5 days prior to surgery, and it was recommended for long-term administration postoperatively. As all 7 patients presented with vertebrobasilar insufficiency, ECA-SV-VA bypass surgery was indicated.

\section{Surgical Technique}

The procedure of exposing and dissecting the $V_{2}$ segment of the VA was performed as described by Bernard George. ${ }^{3,4}$ After general endotracheal anesthesia, the patient was placed in a supine position with the vertex down and the head tilted to the contralateral side approximately $60^{\circ}$. A soft pillow was inserted under the shoulder on the lesion side, and the neck was extended to ease exposure of the ECA and VA. An arc incision along the anterior portion of the sternocleidomastoid muscle was performed.
After dissection and lateral retraction of the sternocleidomastoid muscle, the carotid sheath was exposed and then opened. The ECA trunk and superior thyroid artery were identified and isolated. The carotid sheath was retracted medially, and the longus colli muscle was exposed and dissected. Then, the transverse process was identified, and the vertebral level was confirmed by means of intraoperative fluoroscopy. The anterior part of the transverse process was removed at 1 or 2 levels, and then the VA surrounded by the vertebral venous complex was exposed. The patency of the VA was confirmed, and the blood flow was evaluated by means of intraoperative Doppler flowmetry. Approximately $15 \mathrm{~cm}$ of the SV was harvested with help from cardiac surgeons during the exposure of the VA. After the VA was temporarily trapped by 2 clips and heparin (5000 IU) was administered intravenously, the VA was incised and then anastomosed continuously with the SV graft in a side-to-end fashion, using 6-0 Prolene. Then the other side of the SV graft was anastomosed continuously to the ECA in the same manner. After confirming the patency of the anastomotic stoma by Doppler flowmetry, the blood flow was evaluated again, and then the wound was closed with placement of a subcutaneous drain. The patient was transferred to the intensive care unit, and the endotracheal tube remained in place for 1 day.

\section{Follow-Up Study}

All patients were followed up regularly. NIHSS and $\mathrm{mRS}$ scores were re-evaluated before discharge and monthly during follow-up. TCD and cervical vascular ultrasound were performed at approximately 3 months' follow-up, and CT angiography or cerebral catheter angiography was performed at 6 months' follow-up.

\section{Results}

\section{Findings of Preoperative Angiography}

Preoperative angiography was performed successfully in all cases. Three patients had chronic occlusion in the $\mathrm{V}_{1}$ segment of the dominant VA, and the collateral blood flow arose from the muscular branches of the OA, ascending cervical artery, and deep cervical artery (DCA). In 2 patients with severe in-stent stenosis of the dominant VA, there was no collateral circulation, and the contralateral VA was occluded at the origin or exhibited hypoplasia with distal occlusion. The other 2 patients exhibited an occlusion of the left ScA with retrograde blood flow in the VA (Table 1). Although an interventional revascularization of the ScA was attempted, it failed because the microwire could not pass through the occluded portion.

\section{Surgical Results}

All surgical procedures were performed without intraoperative complications. Five left VAs and 2 right VAs were revascularized. The postoperative course was uneventful in 5 cases, and 2 patients experienced minor complications. Our first ECA-SV-VA bypass patient developed laryngeal edema causing severe respiratory distress, ultimately resulting in apnea and reintubation. The patient recovered uneventfully after re-intubation and steroid treatment. In subsequent procedures, we used soft retraction 
TABLE 1. Summary of demographic and clinical characteristics of the patients in this study

\begin{tabular}{|c|c|c|c|c|c|c|c|c|c|c|c|}
\hline \multirow[b]{2}{*}{$\begin{array}{l}\text { Case } \\
\text { No. }\end{array}$} & \multirow[b]{2}{*}{$\begin{array}{c}\text { Age } \\
\text { (yrs), } \\
\text { Sex }\end{array}$} & \multirow[b]{2}{*}{$\begin{array}{c}\text { Clinical } \\
\text { Presentation }\end{array}$} & \multirow[b]{2}{*}{$\begin{array}{l}\text { Op } \\
\text { Side }\end{array}$} & \multicolumn{3}{|c|}{ Preop DSA } & \multicolumn{3}{|c|}{ Surgical Data } & \multicolumn{2}{|c|}{ Postop Course* } \\
\hline & & & & Lesion & $\begin{array}{l}\text { Collat } \\
\text { Circ }\end{array}$ & Contralat VA & CLA & $\begin{array}{l}\mathrm{EBL} \\
(\mathrm{ml})\end{array}$ & $\begin{array}{l}\text { Occl } \\
\text { Time } \\
\text { (mins) }\end{array}$ & Complication & $\begin{array}{c}\mathrm{FU} \\
\text { (mos) }\end{array}$ \\
\hline 1 & $62, \mathrm{M}$ & Postural dizziness & $\mathrm{Lt}$ & Lt VA occl & $\mathrm{ACA}, \mathrm{OA}$ & Hypoplasia w/ distal occl & $\mathrm{C} 4$ & 360 & 35 & $\begin{array}{l}\text { Apnea on } \\
\text { POD } 1\end{array}$ & 78 \\
\hline 2 & $69, \mathrm{M}$ & $\begin{array}{l}\text { Vertigo, dysarthria, } \\
\text { ataxia }\end{array}$ & Rt & Rt VA occl & ACA & Occl at origin & C5 & 130 & 27 & No & 63 \\
\hline 4 & $59, \mathrm{~F}$ & Vertigo, ataxia & Lt & Lt VA in-stent stenosis & No & Occl at origin & $\mathrm{C} 4$ & 75 & 23 & No & 37 \\
\hline 5 & $51, \mathrm{M}$ & Postural dizziness & $\mathrm{Lt}$ & Lt VA in-stent stenosis & No & Hypoplasia w/ distal occl & C5 & 110 & 28 & No & 31 \\
\hline 6 & $67, \mathrm{~F}$ & Dizziness & $\mathrm{Rt}$ & Rt VA occl & $\begin{array}{c}\text { ACA, OA, } \\
\text { DCA }\end{array}$ & Occl at origin & C5 & 190 & 26 & $\begin{array}{r}\text { Rt shoulder } \\
\text { weakness } \\
\text { on POD } 5\end{array}$ & 19 \\
\hline
\end{tabular}

$\mathrm{ACA}=$ ascending cervical artery; $\mathrm{CLA}=$ cervical level of anastomosis; collat circ = collateral circulation; contralat = contralateral; $\mathrm{DCA}=$ deep cervical artery; $\mathrm{EBL}=$ estimated blood loss; FU = follow-up; mins = minutes; $\mathrm{nrml}=$ normal; occl = occlusion; $\mathrm{POD}=$ postoperative day; $\mathrm{ScA}=$ subclavian artery; $\mathrm{VA}=$ vertebral artery.

* There were no further ischemic events, and angiography demonstrated patent bypasses in all cases.

of the cervical tissue, shortened the operation time, and postponed removal of the endotracheal tube, and the problem did not occur in any of the other 6 cases. One patient complained of weakness in the shoulder on the surgically treated side on postoperative day (POD) 5 and had recovered by POD 14 after rehabilitation. Swelling of the C5 and C6 nerve roots was suspected to underlie the shoulder weakness.

During the operation, the VA occlusion time was 2335 minutes (mean 27 minutes). The recorded blood loss ranged from 63 to $360 \mathrm{ml}$ (mean $154 \mathrm{ml}$ ). Intraoperative Doppler flowmetry showed improved blood flow in the posterior circulation after completion of the bypass. Retrograde flow reversed and became antegrade in the 2 patients with ScA occlusion. The flow velocity of the $V_{2}$ segment was improved in the other 5 patients. All patients were discharged rapidly with close follow-up (Table 1).

\section{Follow-Up}

Ischemic symptoms, such as vertigo and postural dizziness, improved immediately after surgery. Ataxia, hemiparesis and dysarthria improved rapidly after rehabilitation. The duration of follow-up ranged from 12 to 78 months (mean 41.3 months). No patient experienced ischemia of the posterior circulation during the follow-up period. No fresh ischemic lesions were observed on postoperative MRI. The mean NIHSS and mRS scores at the 1-month follow-up examination were 2.85 and 0.86 , respectivelyshowing obvious improvement in comparison to the preoperative scores. In the 3-month follow-up examination, cervical vascular ultrasound confirmed the patency of the anastomosis, and TCD examination showed improvements of flow velocity in the basilar artery and a decreased pulsatility index in the distal VA and basilar artery, which indicated improvement of blood supply in posterior circulation after surgery. Cerebral angiography was performed in 4 patients, and CT angiography was performed in 3 patients at 6 months' follow-up. The images demonstrated patent anastomosis in all cases. In the 2 patients with ScA occlusion, the retrograde blood flow of the VA reverted to normal (Fig. 1).

\section{Illustrative Case}

This 59-year-old woman (case 4) was admitted to our hospital because of symptomatic in-stent stenosis. Two years previously, she was admitted to another hospital because of dizziness. Digital subtraction angiography (DSA) showed occlusion of the right VA at its origin (Fig. 2A) and severe stenosis in the $\mathrm{V}_{1}$ segment of the left VA (Fig. 2B). The stenosis was successfully treated with stent placement (Fig. 2C). One month prior to admission to our hospital, the patient presented to the other hospital with vertigo and ataxia. MRI showed a minor infarction in the brainstem (Fig. 2D), and DSA revealed in-stent restenosis in the left VA (Fig. 2E). After an attempt of in-stent balloon dilation failed, the patient was referred to our hospital, where an ECA-SV-VA bypass was performed (Fig. 3A-E) (Video 1).

VIDEO 1. Video clip showing the surgical procedure of the left ECASV-VA bypass in case 4. Copyright Department of Neurosurgery, Peking University First Hospital. Published with permission. Click here to view.

The patient recovered uneventfully, and postoperative CT angiography showed that the bypass was patent (Fig. 3F). She had no further ischemia events during follow-up.

\section{Discussion}

Symptomatic steno-occlusive diseases of the vertebrobasilar vasculature carry a poor prognosis and high risk of stroke recurrence despite medical therapy. In a previous report, the annual stroke recurrence rates in patients on medical therapy were $7.8 \%$ for stroke in the territory of a stenotic vertebral artery and $10.7 \%$ for stroke in the territory of a stenotic basilar artery.$^{19}$ As the risk of stroke recurrence is much higher for patients with posterior circu- 


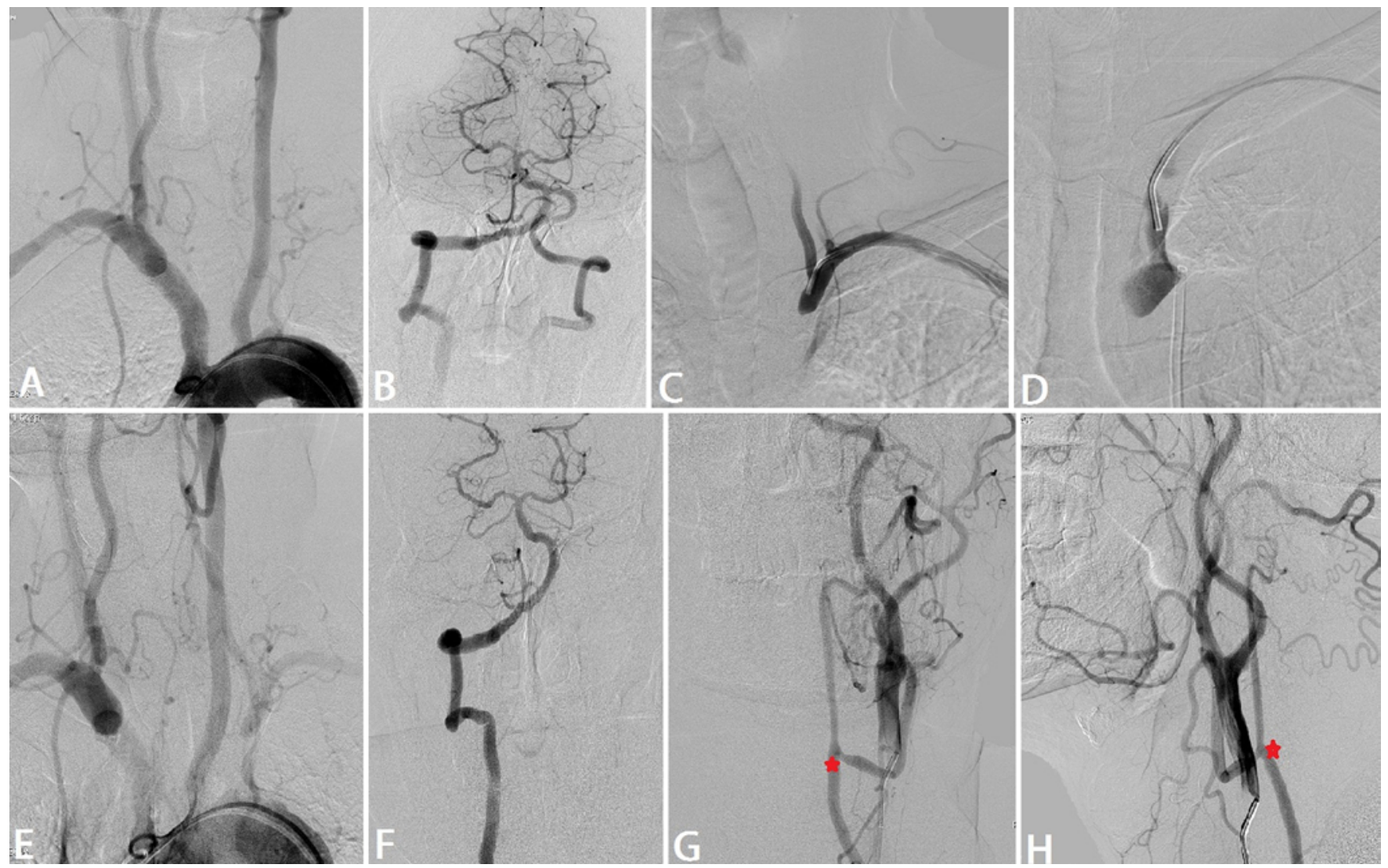

FIG. 1. DSA images showing preoperative (A-D) and postoperative $(E-H)$ blood flow in case 3 . A: Arcus aortae angiography showing occlusion at the origin of the left ScA. B: Right VA angiography showing retrograde blood flow in the left VA. C: Left VA angiography from the radial artery showing the patency of the $V_{1}$ segment and occlusion of the ScA. D: Revascularization of the left ScA through a radial artery and a femoral artery was attempted but stopped when an arcus aortae dissection was noticed. E: Arcus aortae angiography after bypass surgery showing the left ScA in an early stage. F: Right VA angiography showing anterograde blood flow in the left VA. $\mathbf{G}$ and $\mathbf{H}$ : Anteroposterior $(\mathrm{G})$ and lateral $(\mathrm{H})$ views of the left CCA angiography showing the patent anastomosis $\left({ }^{*}\right)$ and normal-direction blood flow in the distal VA.

lation infarction than for those who have suffered carotid territory strokes, patients who have had vertebrobasilar events require more active preventive treatment. ${ }^{8}$ Endovascular surgery has a strong inhibitory effect on stroke recur- rence during acute stages. However, for in-stent restenosis patients or patients with chronic occlusive vertebrobasilar disease, such as VA occlusion and ScA occlusion (subclavian steal syndrome), endovascular treatment is difficult
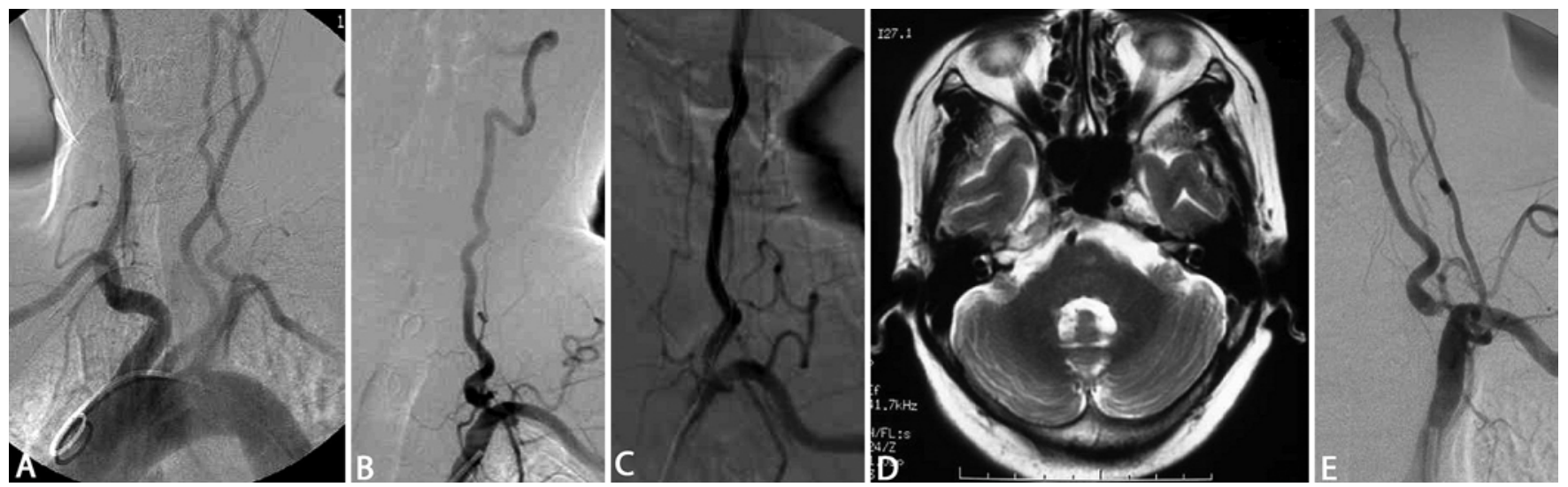

FIG. 2. Case 4. Development of the patient's disease. A: DSA image showing that the right VA is occluded at origin. B: DSA image showing severe stenosis in the $V_{1}$ segment of the left VA. C: DSA image showing resolution of stenosis after stent implantation. D: Axial T2-weighted MR image showing minor infarction in the pons. E: DSA image showing severe in-stent restenosis. 

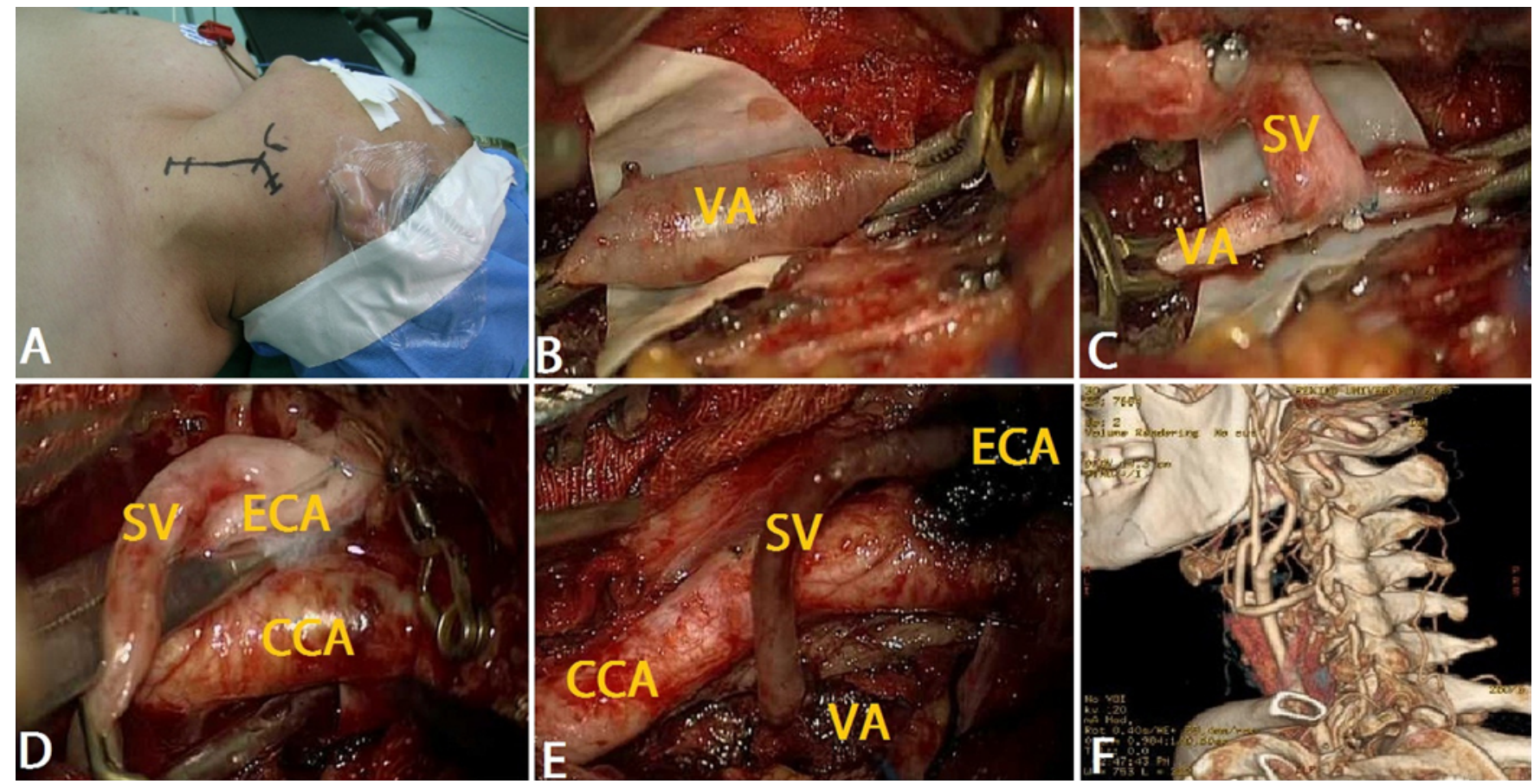

FIG. 3. Case 4. Surgical procedure. A: Surgical position and incision. B: After the transverse foramen is unroofed, the VA is exposed and temporarily occluded by 2 clips. C: Completed end-to-side SV-VA anastomosis. D: Completed end-to-side ECA-SV anastomosis. E: The temporary clips are removed and blood flow from the ECA to the VA is confirmed. F: 3D reconstruction of postoperative CT angiography showing the patency of the bypass.

and not recommended. Nevertheless, for these patients, bypass surgery can be a good choice?

\section{Indications}

Kakino et al. concluded that the ECA-cervical VA $\left(\mathrm{V}_{2}\right.$ segment) bypass with an interposed SV graft is useful "when the VA is occluded at the origin and the distal portion of the cervical VA is perfused via muscular collateral vessels" in patients with medically refractory ischemic events in the vertebrobasilar territory. ${ }^{10}$ However, we found that an ECA-SV-VA bypass was also a good choice for these posterior circulation ischemic patients who still had some VA blood flow, although small or retrograde, such as patients with VA in-stent restenosis and subclavian steal syndrome. In clinical practice, the rate of in-stent restenosis 5 years after percutaneous coronary interventions has been reported to be nearly $5 \%$ for patients treated with drug-eluting stents and $10 \%$ for patients treated with bare metal stents. ${ }^{5}$ For the VA, the incidence varies among different studies from $0 \%$ to more than $50 \%$ due to heterogeneity in study designs, types of stents, follow-up times, and evaluation methods. ${ }^{9}$ The incidence of symptomatic severe in-stent restenosis is unknown, but, in such patients, it is very difficult to perform balloon dilation or a second stent placement in the area with restenosis, as the first stent sometimes protrudes to the lumen of the ScA for approximately 2-3 $\mathrm{mm}$ and forms a sharp angle with the ScA. Endovascular therapy is not effective in these cases, but as long as the VA is patent distal to the stenotic region, there are surgical options. Some surgeons have performed the transposition of the VA to the common carotid artery
(CCA). ${ }^{6}$ This maneuver is reasonable and simple; however, it requires dissecting the cervical VA over 2 or more vertebral segments, increasing the risk of damaging the radiculomedullary branches, which will result in spinal cord injury. In some other patients, the collateral vessels were from the OA or ascending cervical artery to $\mathrm{V}_{3}$ segment, and the proximal VA, including the $\mathrm{V}_{2}$ segment, was occluded. Our strategy of ECA-SV-V $\mathrm{V}_{2}$ segment bypass was not suitable for such patients, although some other investigators applied CCA-distal VA ( $\mathrm{V}_{3}$ segment) bypass with an interposed SV and got a good outcome. ${ }^{16}$ However, for patients like ours with a patent $V_{2}$ segment, this maneuver of $\mathrm{CCA}-\mathrm{V}_{3}$ segment bypass might also have several disadvantages, such as cerebral ischemia during clamping of the CCA and the possibility of graft kinking (due to the long length) when rotating the neck. Our procedure of ECASV-VA bypass does not require transposition of the cervical VA or blocking the CCA blood flow and can provide sufficient blood flow from the ECA to the vertebrobasilar territory with a short graft.

Similarly, with subclavian steal syndrome and occlusion in the proximal part of the ScA, endovascular recanalization is risky and difficult. The microwire might be inserted into a false lumen, which leads to a dissecting aneurysm or aortic rupture..$^{14}$ Other treatment strategies for revascularization, such as CCA to distal ScA bypass or contralateral $\mathrm{ScA}$ to distal $\mathrm{ScA}$ bypass, require complex manipulation, temporary occlusion of the CCA, or the creation of a deep anastomosis in the supraclavicular fossa. ${ }^{17}$ ECA-SV-VA bypass does not require blockade of CCA, and it can provide sufficient blood flow to the posterior 
circulation. This procedure is much easier to perform than CCA-ScA bypass or ScA-ScA bypass with graft. To our knowledge, although the technique of bypass is not new, the utility of this technique in the patients with subclavian steal phenomenon due to proximal ScA occlusion or VA in-stent stenosis is rarely reported.

\section{Technique Notes and Pitfalls}

\section{Exposure of the $V_{2}$ Segment}

The $V_{2}$ segment is the portion of the VA between its entry into the transverse foramen of C6 and its exit from the transverse foramen of $\mathrm{C} 2$. In this segment, it is surrounded by the perivertebral venous plexus and enclosed in a periosteal sheath that is continuous with the periosteum of the foramina of the transverse processes. ${ }^{3}$ Along this course, the VA gives rise to multiple muscular branches and radiculomeningeal branches..$^{18}$ Sometimes when the proximal VA is occluded, the muscular branches become enlarged and provide an important blood supply to the spinal cord and posterior circulation, so the preoperative DSA should be reviewed carefully and the collateral branches should be identified and preserved before the bypass procedure. During the operation, the cervical vertebral level should be confirmed by fluoroscopy. ${ }^{10}$ After dissection and retraction of the musculus longus colli, the transverse process can be exposed and then unroofed by high-speed drill. It is important to confirm that the dissection level of the musculus longus colli is lower than the level of collateral muscular branches; otherwise, the distal blood flow will be blocked. The vertebral vein plexus, which always encircles the VA, should be manipulated carefully. This plexus bleeds easily, but it can be coagulated and patiently dissected by bipolar forceps and microscissors to allow $\mathrm{V}_{2}$ segment exposure.

\section{Anastomosis}

Unlike the blood flow of the anterior circulation, which can be assessed by CT perfusion, it is very difficult to evaluate the degree of ischemia in the posterior circulation, and it is difficult to determine how long the VA can be clamped and the blood flow blocked..$^{13}$ Obviously, the shorter the occlusion time, the better the outcome, but the occlusion time also depends on the collateral blood flow. In cases of chronic VA occlusion in the $\mathrm{V}_{1}$ segment with collateral muscular branches far from the SV-VA bypass, the bypass procedure could be performed deliberately (i.e., with less emphasis on speed). In cases of ScA occlusion with retrograde blood flow in the ipsilateral VA, the blood flow in the vertebrobasilar territory would not be influenced when occluding the VA. However, the blood flow of the ipsilateral upper extremity is blocked, and the bypass procedure should be completed in less than 1 hour; this is similar to the practice of releasing the pressure cuff once per hour during operations on an upper extremity. In patients with symptomatic in-stent restenosis in the dominant VA, as the blood flow of the vertebrobasilar territory is completely blocked, the bypass procedure should be performed as quickly as possible. Our experience shows that 30 minutes of VA occlusion with heparinization is safe, and none of the patients in our case series experienced new neurological deficits after surgery. The anastomosis should be performed in an end-to-side manner, and continuous sutures should be used in order to minimize the time spent tying knots. During anastomosis, we prefer to elevate the mean arterial pressure approximately $20 \mathrm{~mm} \mathrm{Hg}$. After deep anastomosis of the SV-VA, the superficial anastomosis of SV-ECA can be performed easily. After suturing the carotid sheath, we employ drainage with vacuum aspiration to prevent hematoma compression of the trachea.

\section{Pitfalls}

During the procedure, several minor details should be monitored. First, preoperative DSA images should be reviewed carefully. Selection of the recipient segment of the VA should satisfy the following conditions: a) the recipient portion should be patent, and b) the recipient portion should be as low as possible to best preserve the muscular collateral vessels. In addition, the identity of the vertebral segment should be confirmed with intraoperative fluoroscopy before beginning to unroof the transverse foramen. Second, in some cases, the OA is the main collateral vessel, so when performing anastomosis of the ECA-SV, the OA should be protected and not clipped. If the OA is temporarily clipped, then anastomosis should be performed in less than 30 minutes, because collateral blood flow to the vertebrobasilar territory is occluded. Third, as there are some venous valves in the SV, the direction of the bypass should receive special attention. It is possible that a radial artery graft might be better for this reason. Finally, we strongly suggest keeping patients intubated for 24 hours after surgery and routine use of steroids because a long retraction time might result in mucosa edema and lead to postoperative apnea as in case 1 in the present series.

The main limitation of this surgery is that the depth and space constraints of the operative field render the procedure technically difficult. Therefore, it is essential that the surgeon be skilled in the bypass technique and have extensive experience performing bypass procedures. With detailed planning and meticulous manipulation, good outcomes, without neurological deficit, can be achieved for these patients.

\section{Conclusions}

The ECA-SV-VA bypass surgery is a useful method for patients who have suffered medically refractory ischemic events in the vertebrobasilar territory and have severe stenosis or occlusion of the proximal portion of the VA or ScA but a patent $V_{2}$ segment of the VA.

\section{Acknowledgments}

This work was supported by the Chinese National Nature Science Foundation (grant no. 81541119).

\section{References}

1. Benet A, Tabani H, Bang JS, Meybodi AT, Lawton MT: Occipital artery to anterior inferior cerebellar artery bypass with radial artery interposition graft for vertebrobasilar insufficiency: 3-dimensional operative video. Oper Neurosurg (Hagerstown) 13:641, 2017

2. Bouslama M, Haussen DC, Aghaebrahim A, Grossberg JA, Walker G, Rangaraju S, et al: Predictors of good outcome af- 
ter endovascular therapy for vertebrobasilar occlusion stroke. Stroke 48:3252-3257, 2017

3. Bruneau M, Cornelius JF, George B: Anterolateral approach to the V2 segment of the vertebral artery. Neurosurgery 57 (4 Suppl):262-267, 2005

4. Bruneau M, Cornelius JF, Marneffe V, Triffaux M, George $\mathrm{B}$ : Anatomical variations of the V2 segment of the vertebral artery. Neurosurgery 59 (1 Suppl 1):ONS20-ONS24, 2006

5. Cai JZ, Zhu YX, Wang XY, Bourantas CV, Iqbal J, Zhu H, et al: Comparison of new-generation drug-eluting stents versus drug-coated balloon for in-stent restenosis: a meta-analysis of randomised controlled trials. BMJ Open 8:e017231, 2018

6. Diaz FG, Ausman JI, de los Reyes RA, Pearce J, Shrontz C, Pak H, et al: Surgical reconstruction of the proximal vertebral artery. J Neurosurg 61:874-881, 1984

7. Inoue T, Tamura A, Tsutsumi K, Saito I, Saito N: Acute to subacute surgical revascularization for progressing stroke in atherosclerotic vertebrobasilar occlusion. Acta Neurochir (Wien) 154:1455-1461, 2012

8. Jenkins JS, Stewart M: Endovascular treatment of vertebral artery stenosis. Prog Cardiovasc Dis 59:619-625, 2017

9. Jiang Y, Xu X, Wen Z, Xu X, Yang L, Liu X: In-stent restenosis after vertebral artery stenting. Int J Cardiol 187:430433, 2015

10. Kakino S, Ogasawara K, Kubo Y, Kobayashi M, Kashimura $\mathrm{H}$, Ogawa A: Symptomatic occlusion at the origin of the vertebral artery treated using external carotid artery-cervical vertebral artery bypass with interposed saphenous vein graft. Surg Neurol 69:164-168, 2008

11. Katsuki M, Yamamoto Y, Wada N, Kakizawa Y: Occipital artery to extracranial vertebral artery anastomosis for bilateral vertebral artery stenosis at the origin: a case report. Surg Neurol Int 9:82, 2018

12. Keser N, Avci E, Soylemez B, Karatas D, Baskaya MK: Occipital artery and its segments in vertebral artery revascularization surgery: a microsurgical anatomic study. World Neurosurg 112:e534-e539, 2018

13. Lima Neto AC, Bittar R, Gattas GS, Bor-Seng-Shu E, Oliveira ML, Monsanto RDC, et al: Pathophysiology and diagnosis of vertebrobasilar insufficiency: a review of the literature. Int Arch Otorhinolaryngol 21:302-307, 2017

14. Liu Y, Zhang J, Gu Y, Guo L, Li J: Clinical effectiveness of endovascular therapy for total occlusion of the subclavian arteries: a study of 67 patients. Ann Vasc Surg 35:189-196, 2016
15. Markus HS, Larsson SC, Kuker W, Schulz UG, Ford I, Rothwell PM, et al: Stenting for symptomatic vertebral artery stenosis: the Vertebral Artery Ischaemia Stenting Trial. Neurology 89:1229-1236, 2017

16. Miyamoto S, Kikuchi H, Nagata I, Akiyama Y, Itoh K, Yamagiwa $\mathrm{O}$, et al: Saphenous vein graft to the distal vertebral artery between $\mathrm{C}-1$ and $\mathrm{C}-2$ using a lateral-anterior approach. Technical note. J Neurosurg 77:812-815, 1992

17. Potter BJ, Pinto DS: Subclavian steal syndrome. Circulation 129:2320-2323, 2014

18. Tubbs RS, Shah NA, Sullivan BP, Marchase ND, Cohen-Gadol AA: Surgical anatomy and quantitation of the branches of the V2 and V3 segments of the vertebral artery. Laboratory investigation. J Neurosurg Spine 11:84-87, 2009

19. The Warfarin-Aspirin Symptomatic Intracranial Disease (WASID) Study Group: Prognosis of patients with symptomatic vertebral or basilar artery stenosis. Stroke 29:13891392,1998

20. Zheng D, Mingyue Z, Wei S, Min L, Wanhong C, Qiliang D, et al: The incidence and risk factors of in-stent restenosis for vertebrobasilar artery stenting. World Neurosurg 110:e937e941, 2018

\section{Disclosures}

The authors report no conflict of interest concerning the materials or methods used in this study or the findings specified in this paper.

\section{Author Contributions}

Conception and design: Mo. Acquisition of data: Duan. Analysis and interpretation of data: Y Zhang. Drafting the article: J Zhang. Study supervision: Li.

\section{Supplemental Information Videos}

Video 1. https://vimeo.com/303258554.

Video Abstract. https://vimeo.com/306821049

\section{Correspondence}

Dapeng Mo: Beijing Tiantan Hospital Affiliated to the Capital Medical University, Beijing, China. dapengmo@126.com. 